\title{
Editorial
}

\section{Sensors in Intelligent Transportation Systems}

\author{
Krzysztof Okarma $\mathbb{D}^{1},{ }^{1}$ Darius Andriukaitis $\mathbb{D D}^{2}$ and Reza Malekian $\mathbb{D}^{3}$ \\ ${ }^{1}$ Department of Signal Processing and Multimedia Engineering, West Pomeranian University of Technology, Szczecin, \\ Szczecin, 70-313, Poland \\ ${ }^{2}$ Department of Electronics Engineering, Kaunas University of Technology, Kaunas, 51368, Lithuania \\ ${ }^{3}$ Department of Computer Science and Media Technology/Internet of Things and People Research Center, Malmö University, \\ Malmö, 20506, Sweden \\ Correspondence should be addressed to Krzysztof Okarma; okarma@zut.edu.pl
}

Received 23 December 2018; Accepted 24 December 2018; Published 25 February 2019

Copyright (C) 2019 Krzysztof Okarma et al. This is an open access article distributed under the Creative Commons Attribution License, which permits unrestricted use, distribution, and reproduction in any medium, provided the original work is properly cited.

Modern Intelligent Transportation Systems (ITS) are strictly related to the use of various types of sensors to allow gathering information about the parameters of vehicles and their motion. Rapid development of video technology and availability of relatively cheap high resolution cameras cause the increased interest in the use of vision sensors mounted inside vehicles as well as over the roads. Another relevant field of research is related to applications of various passive road sensors mounted in the road as well as traffic sensors such as piezoelectric or magnetic MEMS sensors. One of the most relevant recent challenges and trends in the ITS solutions is related to the fusion of data acquired by various types of sensors, including inertial sensors, GPS solutions, and model based estimators, which can be used as the data sources for connected vehicle technologies.

The purpose of this special issue is to bring together the ideas and recent achievements of various research groups specializing in various aspects of the ITS solutions. It contains papers related to newly developed methods and ideas combining the data obtained from various sensors, including cameras and other research projects and works in the area of Intelligent Transportation Systems with an emphasize on innovations based on sensor approach that address challenges in tracking, localization, road and weather prediction, sensor fusion algorithms, road safety, and anomalies detection.

The special issue consists of eight papers accepted after at least two rounds of reviews organized in the following topics and categories: (i) fusion algorithm for multimodal railway images; (ii) predicting pedestrian counts based on fused infrared-visual videos; (iii) neuromorphic vision based multivehicle detection and tracking; (iv) geostatistical investigation for road weather information systems; (v) magnetic loops as the most used traffic sensors in Intelligent Transportation Systems; (vi) anomalies detection of subway passenger flow; and (vii) vehicle localization methods.

The first paper, written by B. Guo et al., presents the novel multimodal railway image registration method based on the matching of SURF features followed by Contourlet transform image fusion algorithm combined with total variation model and local region energy. The proposed method of image fusion, verified for daytime and night image pairs, allowed reduction of the strong disturbance light of torch at night.

The second article submitted by S. Huang et al. is related to fusion of infrared and visible light videos as well. The application of the proposed approach allows the estimation of the number of pedestrians in outdoor scenarios, in particular at the pedestrian waiting areas. It depends on the estimation of the number of pedestrians based on single-source video and the information fusion based on multisource detection results.

The topic of the third accepted submission is the multivehicle detection and tracking using the neuromorphic vision based approach. G. Chen et al. investigated the advantages of the transmission of only local pixel-level changes caused by the movement in a scene at the time they occur and proposed its application for the ITS. The verification of the detection and tracking accuracy is supported with the analysis of limitations of the proposed solution. 
Another paper is devoted to the geostatistical approach to quantitatively analysis of the spatiotemporal variations of the road weather and surface conditions. The reliability and applicability of the proposed approach have been analysed using the exemplary road weather information systems (RWIS) in Alberta, Canada, and may be useful for planning cost-effective RWIS networks.

The next article authored by F.M. Belenguer et al. covers the topic of inductance calculations of double magnetic loops used as traffic sensors in ITS. The authors discussed geometry, construction, operating mode, and three possible ways to calculate their inductance including their influence on the obtained precision.

The sixth paper deals with the subject of subway passenger flow analysis for anomalies detection using the robust PCA model. The improved anomaly detection method proposed by X. Wang et al., utilizing low-rank nature of the passenger flow data and the sparsity of anomaly data, has been verified experimentally using the real flow data of Beijing subway.

The two last papers correspond with vehicle localization methods using Interval Constraint Propagation and particle filters. The solution proposed by Z. Wang and A. Lambert, originating from the navigation of mobile robots, allows determination of consistently the vehicle's position and orientation by fusing low-cost sensors and map data obtained when a vehicle embedding expensive sensors navigates around the environment. The method has been validated in outdoor scenario for a vehicle equipped with odometers, gyro, and monocular camera.

The method described by K.J. Ahn and Y. Kang utilizes 2D laser range finder measurements and road features, such as curbs, lane lines, and other road markings, for the navigation of autonomous vehicles in urban areas. The authors proposed the application of particle filter for the estimation of the accurate position of a vehicle based on the map of the road features which may be further combined with data from other sensors, including cameras.

\section{Conflicts of Interest}

The guest editors declare that there are no conflicts of interest regarding the special issue.

\section{Acknowledgments}

The guest editors would like to thank the reviewers for the time and efforts they have devoted to provide detailed comments, advice, and suggestions for improvement of the technical and scientific level, as well as the presentation quality of the accepted papers.

Krzysztof Okarma Darius Andriukaitis Reza Malekian 


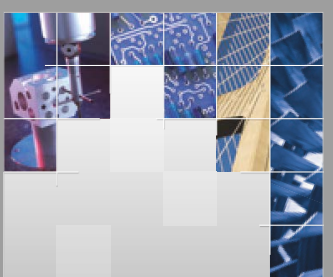

\section{Enfincering}
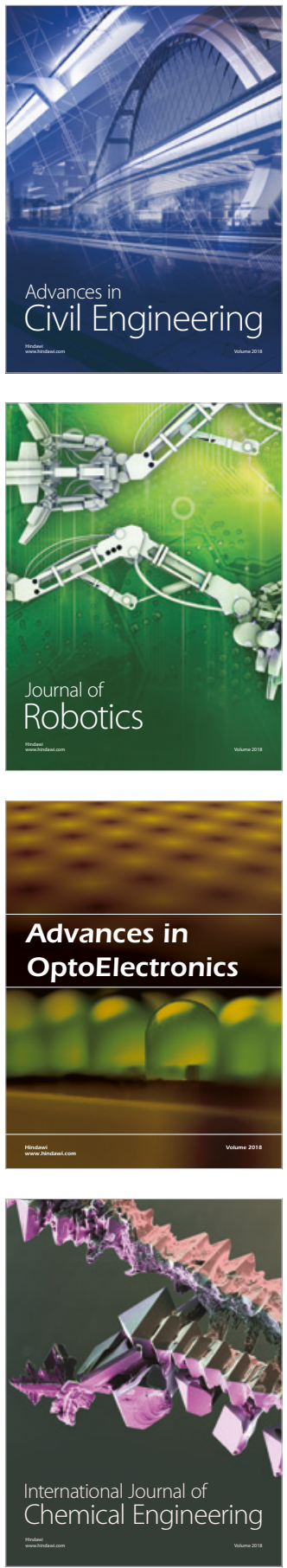

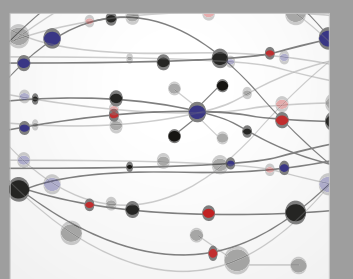

\section{Rotating \\ Machinery}

The Scientific World Journal

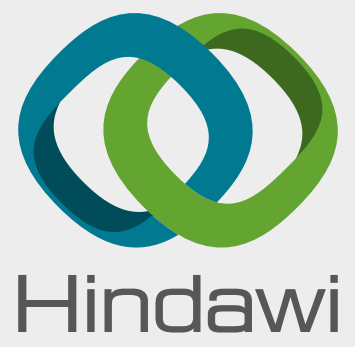

Submit your manuscripts at

www.hindawi.com
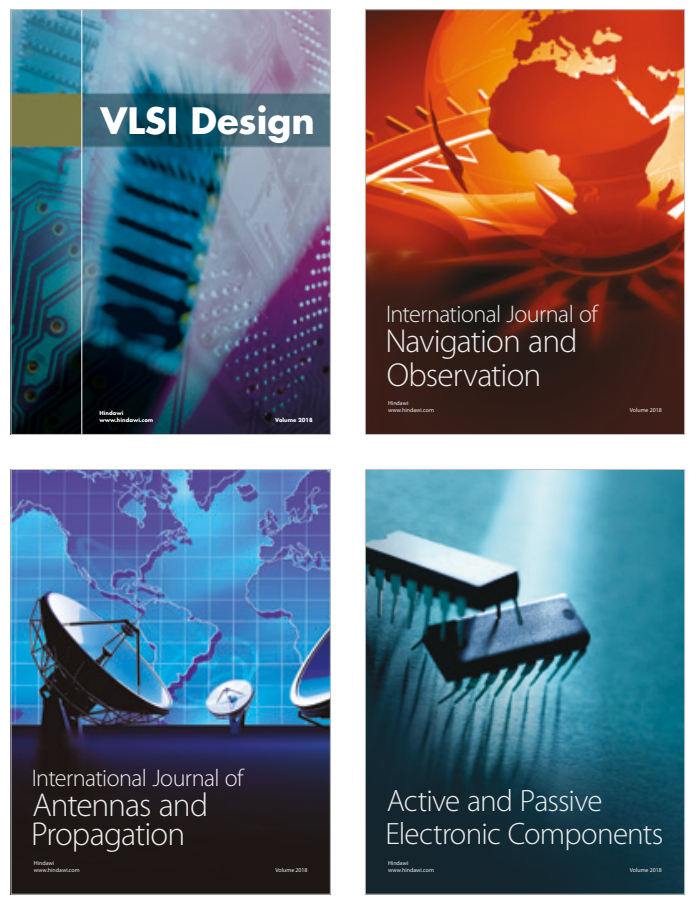
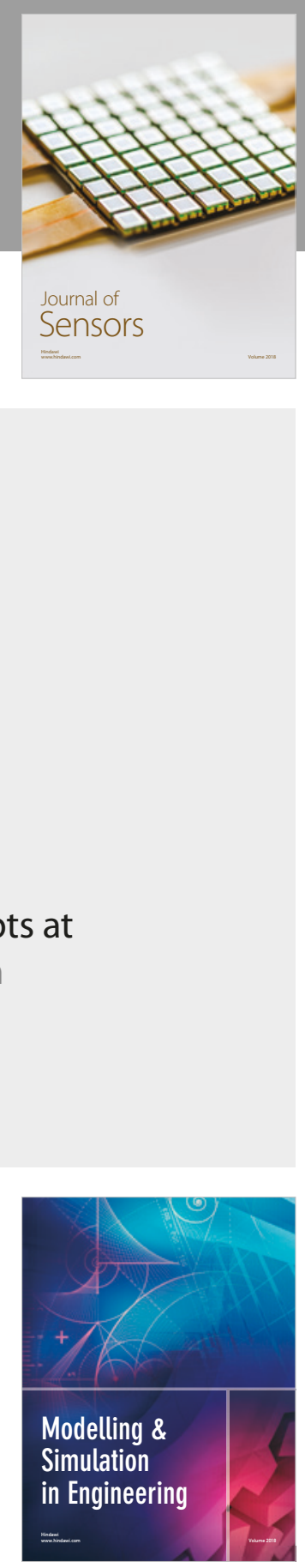

\section{Advances \\ Multimedia}
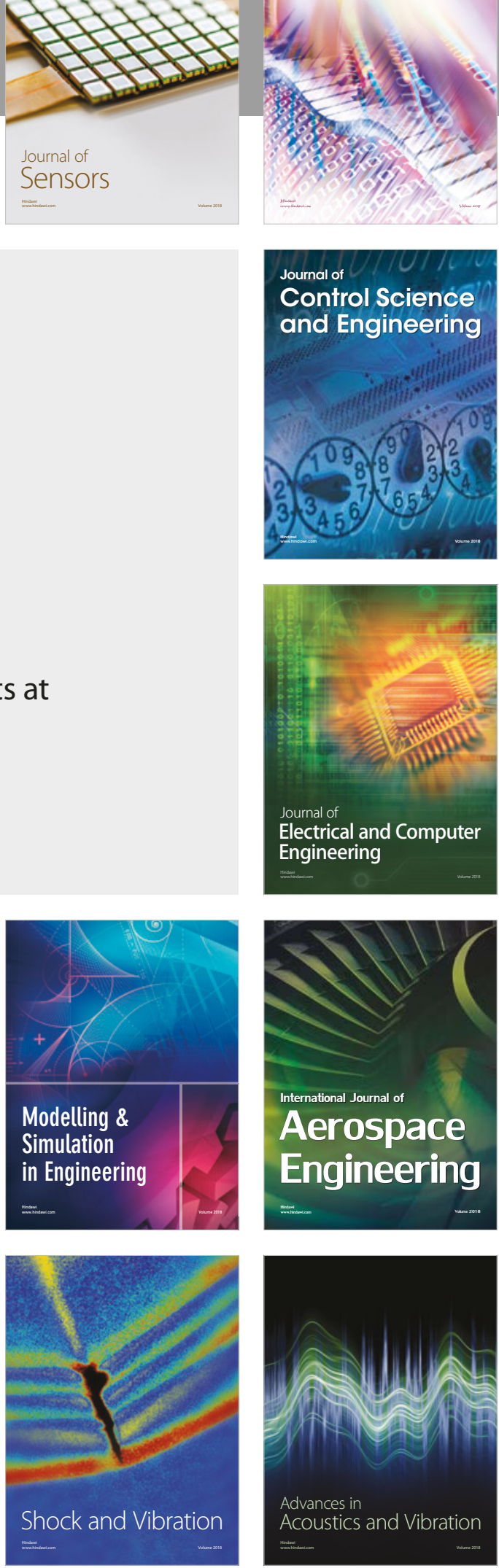